\title{
Fibrant Presheaves of Spectra and Guillén-Navarro Extension
}

\author{
LloRenç Rubió I Pons
}

ABSTRACT - In this remark we prove that the Guillén-Navarro extension of a presheaf of spectra in the category of algebraic varieties over a field of characteristic zero, when exists, coincides up to weak equivalence with the fibrant replacement of the presheaf in the injective model category structure with the cdtopology of abstract blow-ups.

\section{Introduction.}

Guillén and Navarro give in [4] an extension criterion for functors from smooth varieties and taking values in a cohomological descent category. In a cohomological descent category a notion of acyclic square is defined. We have proved [10] (see also [9]) that the category of fibrant spectra has a cohomological descent category structure. In this structure an acyclic diagram of spectra is a homotopy cartesian diagram of spectra.

The extension criterion requires the functor to have a descent property with respect to blow-up squares, i.e. the functor has to carry blow-up squares to acyclic squares in the descent category.

The extended functor is defined for all varieties and satisfies the descent property with respect to abstract blow-ups, which are carte-

(*) Indirizzo dell'A.: Departament de Matemàtica Aplicada 1, Universitat Politècnica de Catalunya, EPSEB, Av. Doctor Marañón, 44-50, 08028 Barcelona, Spain.

E-mail: llorenc.rubio@upc.edu

2000 Mathematics Subject Classification. Primary: 14F20; Secondary: 19D99.

Supported by project MTM2006-14575 (Ministerio de Ciencia y Tecnología) and 2005SGR00557 (AGAUR, Generalitat de Catalunya) 
sian squares

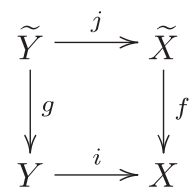

of schemes where $i$ is a closed immersion, $f$ is a proper morphism and the induced morphism $\widetilde{X} \backslash \widetilde{Y} \longrightarrow X \backslash Y$ is an isomorphism. The extended functor is defined using the theory of cubical hyperresolutions [3], and is essentially unique with the descent property.

The extension criterion can also be applied if the functor is already defined for all varieties. Therefore Guillén-Navarro extension criterion can be applied to functors $F$ from schemes to spectra which satisfy a descent condition with respect to smooth blow-up squares, giving a descent functor $F D$.

A functor $F$ from schemes to spectra is also called a presheaf of spectra. The class of abstract blow-up squares defines a $c d$-structure in the sense of Voevodsky [12], and therefore a Grothendieck topology in the category of schemes. We denote $a b s$ this topology. We consider the model category structure of the category of presheaves of spectra studied by Jardine in [6] and [7], where weak equivalences depend on the topology. Given $F$ a presheaf of spectra we denote by $F^{a b s}$ a fibrant replacement in this model category.

Properties of fibrant presheaves make it natural to ask if the descent functor $F D$ obtained from a presheaf of spectra $F$, when exists, coincides with the $a b s$ fibrant replacement $F^{a b s}$.

We prove that when $F D$ is defined, it is globally weakly equivalent to $F^{a b s}$. We also study in which cases $F D$ coincides with $F^{c d h}$, the fibrant replacement with the $c d h$-topology, and obtain a new criterion for $c d h$-descent.

\section{The Guillén-Navarro extension theorem.}

In this section we recall the Guillén-Navarro extension criterion and fix some notations.

The extension criterion of Guillén and Navarro [4] permits to extend a functor from the category of smooth varieties with values in a cohomological descent category and satisfying a descent condition to a functor defined for all varieties. 


\section{1 - Cohomological descent categories.}

Cohomological descent categories [4, Définition (1.5.3) and (1.7.1)] are a variation of triangulated categories, and consist of a cartesian category $\mathcal{D}$ with a saturated class of morphisms $E$ and a simple functor $s$ from cubical diagrams of objects of $\mathcal{D}$ to $\mathcal{D}$, subject to a list of compatibility conditions. An object of $\mathrm{HoD}$ is acyclic if $X \rightarrow 1$ is from $E$, where 1 is the terminal object of $\mathcal{D}$.

The main example of cohomological descent category is the category of chain complexes in an abelian category. Chain complexes in an additive category also have a cohomological descent category structure.

If $\mathcal{D}$ is a descent category, we denote by HoD the localization of $\mathcal{D}$ with respect to the saturated class of morphisms.

We denote by $\mathbf{S p}$ the category of fibrant spectra, in the sense of [1]. We have proved [10] (see also [9]) that stable simplicial model categories are cohomological descent categories with weak equivalences as saturated class of morphisms and homotopy limit as simple functor. In particular this applies to the category $\mathbf{S p}$ of fibrant spectra.

\section{2 - Acyclic squares.}

We fix $k$ a field of characteristic zero. We denote by $\operatorname{Sch}(k)$ the category of reduced separated schemes of finite type over $k$, which we simply call algebraic varieties, and we denote by $\mathbf{S m}(k)$ the category of smooth varieties.

Let

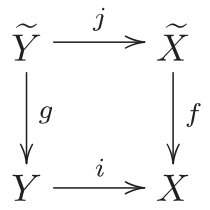

be a cartesian diagram of schemes. Following [4] we say that it is an acyclic square if $i$ is a closed immersion, $f$ is a proper morphism and the induced morphism $\tilde{X} \backslash \tilde{Y} \longrightarrow X \backslash Y$ is an isomorphism. An acyclic square is called in [2] an abstract blow-up.

We say that an acyclic square is an elementary acyclic square if all schemes in the diagram are irreducible and smooth, and $f$ is the blow-up of $X$ along $Y$. 
2.3 - Extension criterion.

The extension criterion is stated for $\Phi$-rectified functors $G: \operatorname{Sm}(k) \rightarrow$ $\rightarrow \mathrm{HoD}$. We recall that the rectification $[4,(1.6 .1)]$ permits to substitute a diagram $G\left(X_{\bullet}\right)$ in $\mathrm{HoD}_{0} \mathcal{D}$, obtained from a diagram $X_{\bullet}$ in $\mathbf{S m}(k)$, by a diagram $G_{\Phi}\left(X_{\bullet}\right)$ in $\mathcal{D}$ in a way such that for every cubical diagram $X_{\bullet}$ of $\operatorname{Sch}(k)$, the object $s G_{\Phi}\left(X_{\bullet}\right)$ of HoD is well defined.

If $G: \mathbf{S c h}(k) \rightarrow \mathrm{HoSp}_{\mathbf{p}}$ is a contravariant $\Phi$-rectified functor and $X_{\bullet}$ is a square in $\mathbf{S c h}(k)$, observe that $\boldsymbol{s} G\left(X_{\bullet}\right)$ is acyclic if and only if $G_{\Phi}\left(X_{\bullet}\right)$ is a homotopy cartesian square of spectra.

Theorem 2.1 ([4], Théorème (2.1.5)). Let D be a cohomological descent category and

$$
G: \operatorname{Sm}(k) \longrightarrow \mathrm{HoD}
$$

a contravariant $\Phi$-rectified functor satisfying the following conditions:

(F1) $G(\emptyset)=1$, and the canonical morphism $G(X \sqcup Y) \longrightarrow G(X) \times G(Y)$ is an isomorphism,

(F2) if $X_{\bullet}$ is an elementary acyclic square in $\mathbf{S m}(k)$, then $\boldsymbol{s} G\left(X_{\bullet}\right)$ is acyclic.

Then there is an extension of $G$ to a $\Phi$-rectified functor

$$
G D: \mathbf{S c h}(k) \longrightarrow \mathrm{HoD}
$$

which satisfies the descent condition

(D) if $X_{\bullet}$ is an acyclic square in $\mathbf{S c h}(k), \boldsymbol{s} G D\left(X_{\bullet}\right)$ is acyclic.

Moreover, this extension is essentially unique: if $G^{\prime}$ is another extension of $G$ verifying the descent property $(D)$, then there is a uniquely determined isomorphism of $\Phi$-rectified functors $G D \Rightarrow G^{\prime}$.

We say that the functor $G D$ has been obtained from $G$ by cubical descent.

The proof of Guillén-Navarro's theorem gives more than stated above. In fact, if $X$ is an algebraic variety and $X \bullet \longrightarrow X$ is any cubical hyperresolution [3], then, under the hypothesis of the theorem,

$$
G D(X)=s G\left(X_{\bullet}\right),
$$

gives a well defined functor from $\operatorname{Sch}(k)$ to $\mathrm{HoD}$, independent of the chosen hyperresolution $X$. 
If the functor $G$ in theorem 2.1 is already defined for all varieties, that is, we have $G: \mathbf{S c h}(k) \longrightarrow \mathrm{HoD}$, and satisfies (F1) and (F2), then there is a natural transformation of $\Phi$-rectified functors $G \Rightarrow G D$.

\section{Presheaves of spectra and $c d$-topologies.}

In this section we recall, fixed a Grothendieck category $\mathcal{C}$ with topology $t$, the model category structure in the category of presheaves of spectra in $\mathcal{C}$ studied by Jardine. We also recall the Grothendieck topologies given by $c d$-structures defined by Voevodsky and the standard $c d$-structures on categories of schemes.

3.1 - Local injective model category structure of presheaves of spectra.

We recall the definitions of global and local weak equivalences of presheaves of spectra, and the model category structure studied by Jardine in [6] and [7].

In this section we fix $\mathcal{C}$ a Grothendieck site with topology $t$, and denote by $\operatorname{Pre}(\mathcal{C}, \mathbf{S p})$ the category of presheaves of spectra in $\mathcal{C}$, i.e. the category of contravariant functors from $\mathcal{C}$ to spectra.

A global weak equivalence of presheaves of spectra is a morphism $f: E \rightarrow E^{\prime}$ such that $E(U) \rightarrow E^{\prime}(U)$ is a weak equivalence of spectra for all objects $U$. Observe that the topology of $\mathcal{C}$ does not intervene in the notion of global weak equivalence. A global cofibration is defined analogously.

A local weak equivalence of presheaves of spectra is a morphism $f: E \rightarrow E^{\prime}$ such that $f$ induces an isomorphism

$$
a_{t} \pi_{*}(E) \longrightarrow a_{t} \pi_{*}\left(E^{\prime}\right)
$$

of sheaves of stable homotopy groups, where $a_{t} F$ denotes the sheaf associated to a presheaf $F$.

Jardine proves [6] [7] that the category $\operatorname{Pre}(\mathcal{C}, \mathbf{S p})$ has a model category structure with local weak equivalences and global cofibrations. In [2, § 3] this structure is called the local injective model category structure.

In the category $\operatorname{Pre}(\mathcal{C}, \mathbf{S p})$ of presheaves of spectra in the site $\mathcal{C}$ with topology $t$ we fix a functorial fibrant replacement $E \longrightarrow E^{t}$ for the local injective model category structure.

Following [2, definition 3.3] we say that a presheaf of spectra $F$ is quasifibrant if the fibrant replacement $F \rightarrow F^{t}$ is a global weak equivalence. 
An important property of fibrant presheaves is that a local weak equivalence between fibrant presheaves of spectra is a global weak equivalence [7] (see also [8, Proposition 3.17]). Observe that this implies that a fibrant presheaf of spectra is quasifibrant.

\section{2 - The cd-structures and associated topologies.}

We recall the definition of $c d$-structure given by Voevodsky in [12] and of its associated topology.

Let $\mathcal{D}$ be a category with initial object. A cd-structure in $\mathcal{D}$ is a class $\mathcal{P}$ of commutative squares closed under isomorphism. The squares in the class are called distinguished squares.

A $c d$-structure in $\mathcal{D}$ defines a Grothendieck topology in $\mathcal{D}$ (see [12, Section 2]).

Following [2] we say that a presheaf of spectra $E$ has the $M V$-property for a family of squares $\mathcal{P}$ if $E$ transforms squares of $\mathcal{P}$ to homotopy cartesian squares of spectra. For example, the (D) property of descent of theorem 2.1 is the $M V$-property with respect to abstract blow-ups.

Voevodsky defines in [12, Section 2] the notions of complete, bounded and regular $c d$-structure. The complete, bounded and regular $c d$-structures have good properties in relation to the model category structure of presheaves, as shown by the following result.

Proposition 3.1. Let $\mathcal{C}$ be a category with a complete, bounded and regular cd-structure $\mathcal{P}$. A local weak equivalence between presheaves that satisfy the MV-property for $\mathcal{P}$ is a global weak equivalence.

Proof. This result for simplicial presheaves is [12, lemma 3.4]. In the proof of [2, theorem 3.4] this result is extended for presheaves of spectra.

The following result, proved by Voevodsky for simplicial presheaves (see [12]), states that under certain conditions the presheaves which satisfy the $M V$-property are precisely the quasifibrant ones.

Theorem 3.2 [2, theorem 3.4]. Let $\mathcal{C}$ be a category with a complete, bounded and regular cd-structure $\mathcal{P}$. Let $E$ be a presheaf of spectra in $\mathcal{C}$. Then $E$ is quasifibrant if, and only if, $E$ has the MV-property for $\mathcal{P}$.

If a presheaf $E$ satisfies the equivalent conditions of this theorem for a topology $t$ generated by a complete, bounded and regular $c d$-structure $\mathcal{P}$ we say, following [2], that $E$ satisfies $t$-descent, or descent for the topology $t$. 
3.3 - The cd-structures in scheme categories.

We recall the standard $c d$-structures on categories of schemes from [13, Section 2].

We consider the following $c d$-structures in the category $\mathbf{S c h}(k)$ :

a) The Nisnevich $\boldsymbol{c d}$-structure where a distinguished square, or elementary Nisnevich square, is a square of the form

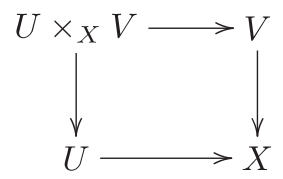

where $U \rightarrow X$ is an open immersion and $V \rightarrow X$ is an étale morphism which is an isomorphism over $X \backslash U$.

The associated topology to the Nisnevich $c d$-structure is the Nisnevich topology (see [13, Proposition 2.16]).

b) The $\boldsymbol{c d}$-structure of abstract blow-ups where the distinguished squares are acyclic squares (see section 2), also called abstract blow-ups.

We denote abs topology the associated topology to the $c d$-structure of abstract blow-ups.

c) The combined $\boldsymbol{c d}$-structure in $\operatorname{Sch}(k)$ is the union of the Nisnevich $c d$-structure and the abstract blow-up structure. It consists of all elementary Nisnevich squares and all acyclic squares.

The associated topology to the combined $c d$-structure is the $c d h$ topology (see [13, Section 2]).

In the category $\mathbf{S m}(k)$ we consider the following $c d$-structures:

$\left.\mathrm{a}^{\prime}\right)$ The Nisnevich $\boldsymbol{c d}$-structure in $\operatorname{Sm}(k)$.

$\left.b^{\prime}\right)$ The $\boldsymbol{c d}$-structure of smooth blow-ups where the distinguished squares are isomorphic to blow-ups of smooth varieties with smooth center.

$\left.c^{\prime}\right)$ The combined $\boldsymbol{c d}$-structre in $\operatorname{Sm}(k)$ is the union of the Nisnevich $c d$-structure and that of the smooth blow-ups. It consists of all elementary Nisnevich squares and the blow-ups of smooth varieties with smooth center.

The topology $s c d h$ is the topology generated by the combined structure in $\operatorname{Sm}(k)$.

EXAMPLE 3.3. Cubical hyperresolutions give coverings in the abs topology formed by smooth varieties. Suppose that $X$. is a 1 -itered cubical 
hyperresolution of $X$ of size $r$ (see [3, Section I.2]). Then we take the varieties with cubical index $\left(\alpha_{0}, \ldots, \alpha_{r}\right)$ with only one $\alpha_{i}$ different of zero.

With the hypotheses of resolution of singularities, which we always consider as we have a field $k$ of characteristic zero, the six cited $c d$ structures are complete, bounded and regular (see [13, Section 2] and the discussion following lemma 4.5 in op.cit.). Therefore we can apply proposition 3.1 and theorem 3.2. We observe in particular the following.

LEMma 3.4. If $F$ is a presheaf of spectra in $\mathbf{S c h}(k), F^{\text {Nis }}$ has the MVproperty with respect to elementary Nisnevich squares, $F^{\text {abs }}$ has the MVproperty with respect to abstract blow-ups and $F^{c d h}$ has the MV-property with respect to the two types of squares.

\section{Comparison of Guillén-Navarro extension with the $a b s$ and $c d h$ fibrant replacement.}

A presheaf of spectra $F$ in $\operatorname{Sch}(k)$ which satisfies $(F 1)$ and $(F 2)$ of theorem 2.1, gives a functor $F D: \mathbf{S c h}(k) \rightarrow$ HoSp. We consider the injective model category structure in the category of presheaves of spectra in $\mathbf{S c h}(k)$ with the abs topology. We have fixed a fibrant replacement $F^{a b s}$ for the presheaf $F$. In this section we prove the main result: $F D(X)$ and $F^{a b s}(X)$ are weakly equivalent for every variety $X$. With extra hypotheses we obtain an analogous result for the $c d h$ fibrant replacement, which permits us to have conditions to assure coincidence between $F^{a b s}$ and $F^{c d h}$.

REMARK 4.1. In fact Guillén-Navarro extension only needs a functor defined for smooth varieties, that is a presheaf of spectra $F$ in $\operatorname{Sm}(k)$. In this case we can extend it to a presheaf of spectra in $\mathbf{S c h}(k)$ by Kan extension and we also consider the fibrant replacements $F^{a b s}$ and $F^{c d h}$ of the extension.

REMARK 4.2. The points of view of Guillén-Navarro extension and the abs fibrant replacement are not always comparable. In Guillén-Navarro's criterion we only need a functor taking values in the homotopy category of spectra, as it is common if in the construction of the functor some kind of resolution take part. As a counterpart, although the input functor $F$ in Guillén-Navarro's criterion can take values in spectra, the descent functor $F D$ always takes values in the homotopy category. 
REMARK 4.3. If $F$ is a presheaf of spectra in $\mathbf{S c h}(k)$ which satisfies the hypotheses of theorem 2.1, then it has the $M V$-property with respect to elementary acyclic squares. Recall that an elementary acyclic square is a blow-up where all varieties are irreductible and smooth. Therefore it has the $M V$-property with respect all smooth blow-ups.

Given a presheaf $F$ in $\mathbf{S c h}(k)$, we denote by $r F$ the presheaf in the subcategory $\mathbf{S m}(k)$ obtained by restriction. The following lemma observes that in the topology abs weak equivalences are conserved by restriction.

Lemma 4.4. Given $f: A \rightarrow B$ a morphism of presheaves of spectra in $\operatorname{Sch}(k)$, if $f$ is a local weak equivalence in the abs topology, then $r f: r A \rightarrow r B$ is a local weak equivalence in the topology of smooth blow-ups.

Proof. It is based on the fact that, with the hypothesis of resolution of singularities, every object of $\mathbf{S c h}(k)$ has an $a b s$-covering by objects of $\mathbf{S m}(k)$, and every abs-covering of an object of $\mathbf{S m}(k)$ has a refinement that is a covering in the topology of smooth blow-ups (see the proof of [13, lemma 4.6]).

LEMMA 4.5. Let F be a presheaf of spectra in $\mathbf{S c h}(k)$ which has the MVproperty with respect to smooth blow-ups. Then given $X$ a smooth variety there is a weak equivalence

$$
F(X) \rightarrow F^{a b s}(X) .
$$

Proof. We reason as in the proof of [2, Theorem 3.12]. By hypothesis $F$ has the $M V$-property for smooth blow-ups and therefore $r F$ has the same property. By the other hand, by theorem 3.2, $F^{a b s}$ has the $M V$-property for smooth blow-ups and therefore $r F^{a b s}$ has the same property. By definition the fibrant replacement $F \rightarrow F^{a b s}$ is a local weak equivalence in the topology $a b s$, and by lemma $4.4, r F \rightarrow r F^{a b s}$ is a local weak equivalence in the topology of smooth blow-ups. We have a local weak equivalence between presheaves that satisfy the $M V$-property, and by proposition 3.1 it is a global weak equivalence in $\operatorname{Sm}(k)$, i.e. $F(X) \rightarrow F^{a b s}(X)$ is a weak equivalence for $X$ smooth.

THEOREM 4.6. Let $F$ be a presheaf of spectra in $\mathbf{S c h}(k)$ which satisfies the hypotheses of theorem 2.1. Then there is a morphism (in HoSp)

$$
F D \longrightarrow F^{a b s}
$$

such that $F D(X) \longrightarrow F^{a b s}(X)$ is a weak equivalence for every variety $X$. 
Proof. By remark 4.3 $F$ has the $M V$-property with respect to smooth blow-ups. By the preceding lemma, $F^{a b s}(X)$ is weakly equivalent to $F(X)$ for smooth $X$. By theorem 3.2, $F^{a b s}$ has the $M V$-property with respect to abstract blow-ups, i.e. $F^{a b s}$ satisfies the (D) property of descent. Therefore, by unicity of Guillén-Navarro extension we have the result.

We can prove an analogue result for the $c d h$ topology. First we observe that in the $c d h$ topology local weak equivalences are also conserved by restriction.

Lemma 4.7 [2, lemma 3.11]. Given $f: A \rightarrow B$ a morphism of presheaves of spectra in $\mathbf{S c h}(k)$, if $f$ is a local weak equivalence in the cdh topology, then $r f: r A \rightarrow r B$ is a local weak equivalence in the scdh topology.

PRoof. It is based on the fact that, with the hypothesis of resolution of singularities, every object of $\operatorname{Sch}(k)$ has a $c d h$-covering by objects of $\mathbf{S m}(k)$, and every $c d h$-covering of an object of $\mathbf{S m}(k)$ has a refinement which is a $s c d h$-covering (see the proof of [13, lemma 4.6]).

To obtain that $F$ and $F^{c d h}$ coincide for smooth varieties it is necessary to suppose that $F$ has the $M V$-property for smooth blow-ups and for smooth Nisnevich squares.

LEMma 4.8. Let $F$ be a presheaf of spectra in $\mathbf{S c h}(k)$ with the MVproperty with respect to smooth blow-ups and smooth elementary Nisnevich squares. Then given $X$ a smooth variety there is a weak equivalence

$$
F(X) \rightarrow F^{c d h}(X) \text {. }
$$

Proof. We reason as in the proof of [2, Theorem 3.12]. By one hand, $F$ has the $M V$-property for smooth elementary Nisnevich squares and for smooth blow-ups and therefore $r F$ has the $M V$-property for the combined structure in $\operatorname{Sm}(k)$. By the other hand, by theorem 3.2, $F^{c d h}$ has the $M V$ property for the combined structure in $\mathbf{S c h}(k)$, and therefore $r F^{c d h}$ has the $M V$-property for the combined structure in $\operatorname{Sm}(k)$. By definition of fibrant replacement $F \rightarrow F^{c d h}$ is a local weak equivalence in the $c d h$ topology, and by lemma $4.7, r F \rightarrow r F^{c d h}$ is a local weak equivalence in the $s c d h$ topology. We have a local weak equivalence between presheaves which satisfy the $M V$-property, and by proposition 3.1 it is a global weak equivalence in $\operatorname{Sm}(k)$, i.e. $F(X) \rightarrow F^{c d h}(X)$ is a weak equivalence for $X$ smooth. 
THEOREM 4.9. Let $F$ be a presheaf of spectra in $\mathbf{S c h}(k)$ which satisfies the hypotheses of theorem 2.1 and the MV-property with respect to smooth elementary Nisnevich squares. Then there is a morphism (in HoSp)

$$
F D \longrightarrow F^{c d h}
$$

such that $F D(X) \longrightarrow F^{c d h}(X)$ is a weak equivalence for every variety $X$.

Proof. By remark 4.3 $F$ has the $M V$-property with respect to smooth blow-ups. By previous lemma, $F^{c d h}(X)$ is weakly equivalent to $F(X)$ for $X$ smooth. By theorem 3.2, $F^{c d h}$ has the $M V$-property with respect to abstract blow-ups, i.e. $F^{c d h}$ satisfies the (D) property of descent. Therefore, by unicity of Guillén-Navarro extension we have the result.

With the hypotheses of this theorem, we have that both $F^{c d h}$ and $F^{a b s}$ are globally weakly equivalent to $F D$, and therefore $F^{c d h}$ and $F^{a b s}$ are weakly equivalent. More precisely we have the following result.

Proposition 4.10. Let $F$ be a presheaf of spectra in $\mathbf{S c h}(k)$ with the MV-property with respect to smooth blow-ups and smooth elementary Nisnevich squares. Then the morphisms

$$
F^{a b s} \longrightarrow\left(F^{c d h}\right)^{a b s} \longleftarrow F^{c d h}
$$

are global weak equivalences.

PRoof. The second morphism is always a global weak equivalence. For the first morphism it is enough to see that $F \rightarrow F^{c d h}$ is a local weak equivalence in the $a b s$ topology, by proposition 3.1 and functoriality of fibrant replacement. Proof of lemma 4.4 shows that its converse is also true, so it is enough to see that $r F \rightarrow r F^{c d h}$ is a local weak equivalence in the topology of smooth blow-ups. But $r F \rightarrow r F^{c d h}$ is a global weak equivalence by lemma 4.8 , and therefore a local weak equivalence.

We can interpret this result as a condition for $c d h$-descent (compare with [2, Theorem 3.12]).

Corollary 4.11. Let $F$ be a presheaf of spectra in $\mathbf{S c h}(k)$ with the MV-property with respect to abstract blow-ups and smooth elementary Nisnevich squares. Then F satisfies cdh-descent.

Proof. As $F$ satisfies descent for abstract blow-ups, by theorem $3.2 F$ 
and $F^{a b s}$ are globally weakly equivalent. By the previous proposition $F^{a b s}$ and $F^{c d h}$ are globally weakly equivalent. It follows that $F$ and $F^{c d h}$ are globally weakly equivalent and therefore $F$ satisfies $c d h$-descent.

\section{Example.}

We apply this results to algebraic K-theory. We let $\mathcal{K}$ denote the presheaf of spectra of non-connective K-theory as defined in [11, Section 6.4].

Proposition 5.1. For every variety $X$ there is a weak equivalence

$$
\mathcal{K}^{a b s}(X) \simeq \mathcal{K}^{c d h}(X) .
$$

Proof. We apply proposition 4.10. For the $M V$-property with respect to smooth blow-ups see [9] for a detailed discussion (see also [2, Remark 1.6]). The presheaf $\mathcal{K}$ also satisfies Nisnevich descent [11, 10.8], so all hypothesis of proposition 4.10 are fulfilled.

Let $\mathcal{K H}$ denote Weibel's homotopy invariant K-theory of [14] as formulated in [11, Section 9.11]. Haesemeyer proves in [5] that $\mathcal{K} \mathcal{H}(X) \simeq \mathcal{K}^{c d h}(X)$ for every variety $X$. Therefore we obtain that also $\mathcal{K} \mathcal{H}(X) \simeq \mathcal{K}^{a b s}(X)$ for every variety $X$.

\section{REFERENCES}

[1] A. K. Bousfield - E. M. Friedlander, Homotopy theory of $\Gamma$-spaces, spectra, and bisimplicial sets. In Geometric applications of homotopy theory (Proc. Conf., Evanston, Ill., 1977), II, volume 658 of Lecture Notes in Math. (Springer, Berlin, 1978), pp. 80-130.

[2] C. Cortiñas - C. Haesemeyer - M. Schlichting - C. Weibel, Cyclic homology, cdh-homology and negative k-theory. Annals of Mathematics, 167 (2008), pp. 549-573.

[3] F. Guillén - V. Navarro Aznar - P. Pascual Gainza - F. Puerta, Hyperrésolutions cubiques et descente cohomologique, volume 1335 of Lecture Notes in Mathematics. Springer-Verlag, Berlin, 1988. Papers from the Seminar on Hodge-Deligne Theory held in Barcelona, 1982.

[4] Francisco Guillén - Vicente Navarro Aznar, Un critère d'extension des foncteurs définis sur les schémas lisses. Publ. Math. Inst. Hautes Études Sci., 95 (2002), pp. 1-91.

[5] Christian Haesemeyer, Descent properties of homotopy K-theory. Duke Math. J., 125, 3 (2004), pp. 589-619.

[6] J. F. JaRdine, Simplicial presheaves. J. Pure Appl. Algebra, 47, 1 (1987), pp. $35-87$. 
[7] J. F. JARDIne, Stable homotopy theory of simplicial presheaves. Canad. J. Math., 39, 3 (1987), pp. 733-747.

[8] Stephen A. Mitchell, Hypercohomology spectra and Thomason's descent theorem. In Algebraic $K$-theory (Toronto, ON, 1996), volume 16 of Fields Inst. Commun., Amer. Math. Soc., Providence, RI (1997), pp. 221-277.

[9] Pere Pascual Gainza - Llorenç Rubió i Pons, Algebraic k-theory and cubical descent. Preprint Arxiv math. AG/2257, june of 2007.

[10] Llorenç Rubió i Pons, Model categories and cubical descent. Bol. Soc. Mat. Mexicana, 13, 3 (2007), pp. 293-305.

[11] R. W. Thomason - Thomas Trobaugh, Higher algebraic K-theory of schemes and of derived categories. In The Grothendieck Festschrift, Vol. III, volume 88 of Progr. Math. (Birkhäuser Boston, Boston, MA, 1990), pp. 247-435.

[12] Vladimir Voevodsky, Homotopy theory of simplicial sheaves in completely decomposable topologies. Preprint (September 6, 2000), K-theory Preprint Archives, http://www.math.uiuc.edu/K-theory/0443/.

[13] Vladimir Voevodsky, Unstable motivic homotopy categories in Nisnevich and cdh-topologies. Preprint (September 6, 2000), K-theory Preprint Archives, http://www.math.uiuc.edu/K-theory/0444/.

[14] Charles A. Weibel, Homotopy algebraic K-theory. In Algebraic K-theory and algebraic number theory (Honolulu, HI, 1987), volume 83 of Contemp. Math., Amer. Math. Soc., Providence, RI (1989), pp. 461-488.

Manoscritto pervenuto in redazione il 5 gennaio 2009. 
